\title{
14. REGISTER
}

\subsection{Personen}

Aalen, Sverre $2^{5}, 499$

Abélès, Luce $389^{96}, 499$

Achten, Udo $448^{297}, 499$

Achterberg, Christoph $8^{34}, 499$

Agard, Charles-Jean $408^{166}, 477$

Agulhon, Maurice $8^{+32}, 159^{59}, 173^{+100}$, $189^{170}, 201^{221}, 234^{31}, 341^{158}, 345^{173}, 499$

Aisenberg, Andrew R. $389^{97}, 391^{99}$, 499

Albert, Pierre $\quad 63^{1}, 68^{31}, 73^{47}, 76^{53}, 499$

Albrecht, Juerg $112^{113}, 125^{153}, 499$

Albrecht, Michael von 494

Alembert, Jean Le Rond d' $31^{+48}, 53$, $335^{139}, 493$

Alewyn, Richard $98^{58}, 499$

Alexander der Große 135

Allendorf, Johannes $209^{251}, 518$

Altkind, Julius 478

Amade, Gérard Léo, General d' 72

Amalvi, Christian $264^{125}, 499$

Ambroise-Rendu, Anne-Claude $337^{148}$, $373^{53}, 499$

Amelunxen, Hubertus von $337^{148}, 499$

Andia, Beatrice de $189^{164}, 513$

Angenot, Marc $397^{119}, 399^{129}, 499$

Anquetin, Louis $290^{14}$

Antoine, J. J. 478

Apostolidès, Jean-Marie $40^{12}, 499$

Arjès, Philippe $169^{95}, 389^{98}, 500,507,508$, 516

Arnoux, Charles-Albert d' 478

Asmodée 477

Astaroth 477

Aubert, Andreas $454^{323}$

Auer von Welsbach, Carl Freiherr 359

Augsburger, Janis $473^{15}, 509$

Augustinus $26^{+10}$

Aurelian $39^{11}$

Avenel, vicomte d' $180^{130}, 358^{16}, 493$

Babinet, Jacques $59^{+119,120}, 83^{84}$

Bachelard, Gaston $52^{81}, 396^{114}, 500$

Bachollet, Raymond $76^{53}, 77^{55}, 78^{66}$, $326^{108}, 500$

Baeque, Antoine de $8,37^{1}, 500$

Bahr, Ehrhard $35^{73}, 493$
Bailly-Herzberg, Janine $113^{116}, 400^{137}$, 495, 500

Bala d'Eche $289^{7}, 477$

Balluriau, Jean-Baptiste 477

Balluriau, Paul $205,317^{+84}, 318,319,320$, 477,490

Bancquart, Marie-Claire $93^{41}, 500$

Barbier, Pierre $140^{202}, 494$

Barbin, Charles $186^{152}, 493$

Bargeton, Delphine $21^{89.91}, 23^{96}, 63^{1}, 500$

Bargiel, Réjane $406^{155}, 434^{250}, 500$

Baric, Jules 123, 124, 478

Barré, Raoul 478

Barrows, Susanna $106^{97}, 132^{183}, 298^{29}$, 500

Barthes, Roland $1^{3}, 16^{+72}, 17^{+73.74 .75}$, $18^{+81}, 245^{68}, 471^{+13}, 500$

Bartholdi, Frédéric Auguste $201^{+221}$, 499

Basile $163^{+75.76}, 180,186,231,235,236$, $259,352+191$

Baubérot, Arnaud $4355^{255.256 .257}, 436^{266}$, $437^{274}, 500$

Baubérot, Jean $171^{97}, 188^{162}, 500$

Baudelaire, Charles $71^{+39}, 385^{80}, 493$

Baudoin, I. $102^{75}, 495$

Bausinger, Hermann $471^{11}, 500$

Bayard, Emile $70^{+36}, 72^{+42}, 262^{114}, 493$

Bayle, Pierre $27^{28}$

Bazetoux, Denise $82^{83}, 400^{137}, 500$

Beaumarchais, Pierre Augustin Caron de $163^{75}$

Bébé 485

Beck, Herbert $34^{70}, 524$

Becker, Colette $306^{55}, 495$

Becker, Karin Elisabeth $25^{3}, 26^{13} \cdot 27^{17.20}$. ${ }^{28}, 31^{44}, 32^{+51}, 33^{57.60}, 33^{63}, 34^{70.71}, 500$

Béguet, Bruno $173^{101}, 500$

Beierwaltes, Werner $25^{2.4}, 26^{10.15}, 27^{19}$, 500

Bellanger, Claude $63^{1}, 68^{31}, 76^{52}, 499$. $500,503,521$

Beller, Manfred $45^{39}, 500$

Bellet, Roger $66^{16}, 500$

Belloguet, André 153,478

Belloquet 478 
Beltran, Alain $176^{108,111}, 178^{122}, 180^{129}$, ${ }^{130}, 194^{188}, 236^{39}, 238^{44,45}, 246^{78}, 358^{15}$, $359^{20}, 362^{28}, 408^{169}, 411^{175,179,180,181}$, $412^{186}, 418^{205}, 422^{216}, 423^{219}, 500$

Ben-Amos, Avner $144^{7}, 191^{179}, 216^{269}$, $266^{134}, 501$

Beneke, Sabine $440^{278}, 509$

Bénézit, Emmanuel $477,478,479,480$, $481,482,483,484,485,501$

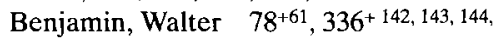
${ }^{145}, 394^{+108}, 456^{+330}, 457,501,502$

Bense, Max $18^{79}, 501$

Benz, Ernst $45^{42}, 501$

Béranger, P.-J. de 53, 185+146, 493, 497

Berger, G. $353^{194}$

Berger, Peter L. $16^{+69}, 501$

Berlanstein, Leonard R. $100^{68}, 176^{108}$ $365^{35,36}, 501$

Bernay, Paul $147^{+16}, 478$

Bernhard von Chartres 31

Bertall, Paul $130^{178}$

Bertrand Dorléac, Laurence $8^{34}, 505$

Berz, Peter $19^{84}, 501$

Bexte, Peter $324^{98,100}, 335^{139}, 501$

Biet, Christian $26^{12}, 501,504,510$

Binczek, Natalie $335^{139}, 501$

Binder, Beate $238^{44}, 240^{+48}, 501$

Bismarck, Otto von 218,220

Blass, Christiane $60^{121}, 501$

Blass, J. $67,68,79^{67}, 135^{193}, 136,161$, $217^{272}, 222,235^{33}, 265,266^{+135}, 267^{139}$, $268,309^{+63}, 478,488,489,501$

Bloch, Charles $87^{9}, 108^{105}, 501$

Bloch, Ernst $453^{312}, 501$

Blondel $217^{275}, 478$

Blondel, Carl-Albert 478

Blühm, Andreas $12^{49}, 61^{127}, 100^{69}, 169^{93}$, $176^{112}, 192^{182,183}, 194^{190}, 229^{15}, 236^{38}$, $240^{49}, 241^{52}, 245^{71}, 250^{91}, 272^{156}, 356^{7}$, $357^{9}, 359^{23}, 367^{41}, 392^{107}, 473^{14}, 501$

Blumenberg, Hans $3^{12}, 7^{28}, 10^{+43}, 17^{72}$, $26^{9}, 28^{30}, 319^{89}, 325^{104}, 327^{112}, 501$

Bobb $82,312^{71}, 478,483$

Böcher, Otto $2^{5}, 3^{10}, 42^{25}, 501$

Boehm, Gottfried $\quad 6^{26,27}, 17^{+76}, 501$

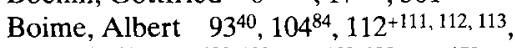
$113^{114,115}, 117^{128,130}, 118^{132,135}, 130^{179}$, $158^{53}, 231^{19}, 234^{31}, 254^{100}, 433^{246}, 502$

Bois, Jean-Pierre $225^{4}, 235^{37}, 256^{105}$, 502

Boisdeffre, Raoul François Charles Le Mouton de $304^{+47}$

Bonaparte, Jérôme $259^{113}$
Bonaparte, Louis-Napoléon, s. auch Napoleon III. $58,226^{10}, 416$

Bonaparte, Napoléon, s. auch Napoleon I. $151,161,181,232$

Bonaparte, Victor $259^{113}$

Bonhomme, Jacques 478

Bonnefous, Georges $208^{243}, 209^{249}, 321^{96}$, $352^{189}, 401^{140}, 502$

Bonnet, Raoul $52^{82}, 502$

Boppe, August $52^{82}, 502$

Bork, Angela $85^{1}, 502$

Bormann, C. von $25^{2}, 26^{10}, 500$

Bosch-Abele, Susanne $55^{95}, 56^{102,103}$, $232^{27}, 502$

Boscovitz, F. $206^{237}$

Bosse, Monika $59^{121}, 157^{47}, 265^{133}, 502$

Boudard, Jean-Baptiste $102^{75}, 495$

Boudon, Jacques-Olivier $196^{198}, 502$

Bouin-Luce, Jean $\quad 82^{83}, 500$

Boukay, Maurice $287^{+173}, 442^{291}, 448^{+301}$, $449^{+304}, 457^{332}, 493$

Boulanger, Georges Ernest Jean Marie, General $72^{45}, 159,160,161,200,242$, $244^{+65}, 259,262^{+116}, 263,388^{90}, 444^{292}$

Bouman, Mark J. 3567,502

Bourdais, Jules $245^{+73}, 493,520$

Bourgevin 478

Boussel, Patrice $287^{2}, 290^{11}, 312^{68}, 502$

Boutigny, Paul 496

Bracco, Patrick $232^{26}, 233^{30}, 502$

Bradberry, Georges $441^{284}$

Braun, Andreas $451^{307}, 502$

Braunthal, Julius $274^{163}, 502$

Brebeck, Christian $64^{5}, 89^{20}, 143^{5}, 502$

Bredin, Jean-Denis $287^{1}, 317^{85}, 321^{92,93}$, ${ }^{95}, 328^{121}, 502$

Bréger, A. E. $203,204,205^{229}, 478,488$

Bremer, Dieter $3^{14}, 25^{5}, 502$

Bréton, L. $105^{86}, 502$

Briand, Aristide $203,208^{+244}, 209,420^{+208}$, 422, 423

Briese, Olaf $45^{43,44}, 47^{56}, 502$

Brisson, Adolphe $70^{36}, 71^{40}, 35^{17}, 493$

Brisson, Henri $326^{+110}$

Broglie, Herzog von $165^{+41}$

Brousset, B. $205,213,379,380,381,478$, 491

Brown, Stephen $288^{4}, 502$

Bruant, Aristide 497

Brullé, Pierre $338^{151}, 339^{153}, 522$

Brunner, Otto $3^{11,507}$

Buchholz, Kai $436^{264}, 455^{323}, 460^{340}, 502$, 522 
Buchholz, Michael B. $325^{103}, 518$

Buchinger-Früh, Marie-Luise $105^{87,88,89}$, 502

Buck-Morss, Susan 502

Buisson, Etienne $410^{170,171}, 411^{179}, 426^{220}$, 493

Bultmann, Rudolf $25^{1}, 502$

Burnand, Robert $139^{+200}, 502$

Bury, John P. T. $89^{17}, 503$

Busch, Bernd $232^{24}, 503,517,523$

Cadel, Eugène $379^{+65}, 478$

Caillebotte, Gustave $113^{+114}, 117,385^{80}$, 478

Caillou, Jacques 82,485

Candide 478

Canetti, Elias $101^{73}, 106^{94}, 503$

Caporal 482

Caran d'Ache $78^{62}, 82^{82}, 312^{71}, 478,497$

Cardon, François $362^{28}, 503$

Cardot, Fabienne $176^{109}, 246^{76}, 362^{28}$, 503, 507

Carnot, Sadi $72,262,399,431$

Caron, François $58^{117}, 60^{123,124}, 6^{128}$, $85^{1,2}, 87^{9}, 91^{70}, 149^{19}, 153^{32.37}, 154^{39}$, $156^{45}, 162^{68,72}, 184^{143}, 212^{258}, 220^{285}$, $262^{115}, 263^{122}, 503$

Caron, Jean-Claude $101^{70}, 104^{83}, 105^{86}$, $107^{99}, 129^{175}, 130^{180}, 503$

Carré, Patrice A. $176^{108.111}, 178^{122}$, $180^{129,} 130,194^{188}, 236^{39}, 238^{45}, 246^{78}$, $358^{15}, 359^{20}, 362^{28}, 408^{169}, 411^{175,} 179,180$, $412^{186}, 422^{216}, 423^{219}, 501$

Carrier, Ed $217^{281}, 478$

Caserio, Jeronimo 399

Casimir-Perier, Jean $\quad 429+231,431$

Cassagnac, Paul de $158^{+55}, 159^{+57.58}, 244^{65}$

Cassirer, Ernst $2^{+5}, 503$

Cate, Phillip Dennis $79^{69} .71,120^{141}$, $270^{151}, 287^{4}, 290^{14}, 302^{40}, 341^{156}, 346^{179}$, $382^{72}, 385^{81}, 405^{155}, 418^{204}, 429^{227}, 448^{300}$, $503,506,521,522$

Cavaignac, Godefroy 326

Céard, Jean $30^{43}, 495$

Célérier, Patricia-Pia 129175, 503

Cémoi 485

Chabanne, Thierry $105^{88}, 309^{62}, 503$

Chalas, Yves $43^{31}, 516$

Cham $67^{+20}, 95,99,101^{71}, 102,103,104$, $105^{+87}, 106,110,111,133^{188}, 164,165,167$, $168,173,178^{122}, 179^{125}, 181,192,194^{190}$, $256^{103}, 367^{41}, 371,372,373,374,382^{72}$, $440,478,487,488,491,492,496,515$
Chambord, Comte de, auch genannt Henri V $259^{113}$

Champfleury $70^{37}, 493$

Changeux, Jean-Pierre $12^{49}, 503$

Chanteclair $82,137^{+195}, 478$

Chapeaurouge, Donat $41^{24}, 45^{41}, 144^{8}$, 503

Chapple, Gerald $460^{340}, 510$

Charle, Christophe $305^{50}, 503$

Charles X, s. Karl X.

Charlet, Louis $76^{52}, 503$

Charmeil, Gilbert $64^{5}, 89^{20}, 502$

Charvic 270, 478

Chastenet, Jacques $85^{1}, 87^{10}, 88^{14}, 89^{18}$, $90^{25}, 108^{106}, 154^{41}, 160^{61}, 161^{64.65}$, $203^{222,223,225}, 220^{283}, 317^{86}, 414^{189}, 503$

Chatenay, Edouard 496

Chaudonneret, Marie-Claude 23431, 503

Chauvaud, Frédéric $117^{127}, 118^{133}, 503$

Chevalier, Louis (Zeichner) 332, 333, 479,490

Chevalier, Louis (Autor) $106^{95}, 355^{2}$, $375^{56}, 503$

Chevalier, Louis-Marie-Jean-Baptiste 479

Chifflard, M. 479

Chifflart, Nicolas-François 479

Choné, Paulette $99^{61}, 169^{95}, 503$

Choubrac, Léon 481

Chouillet, Jacques $33^{65}, 503$

Christian $248^{85}, 249,479,489$

Christian, Paul Marie Bernard 479

Chronos $38,39,40,51^{75}, 110,317^{83}, 324$, $325,326,387^{89}, 448,456^{328}$

Cillessen, Wolfgang $45^{40}, 50^{71}, 504$

Claeys, Gregory $432^{240}, 504$

Classen, C. J. $25^{1}, 504$

Clayson, Hollis $\quad 97^{47}, 105^{87}, 504$

Clemenceau, Georges $161,203,312^{71}$, $313^{72}, 314,316^{77}, 344^{+165}, 412,413,416$, 491

Clément, Jean 479

Clément, Jean-Jacques 479

Clésinger, Jean-Baptiste dit Auguste $233^{+31}, 234^{+31}, 235,479$

Cochin, Charles Nicolas le jeune $102^{75}$, $140,324,325^{103}, 479,495$

Coco 483

Cohl, Emile $82^{+82}, 130,131,236,237$, $259^{+111}, 260,309^{62}, 478,479,487,489$, 504

Collier, Peter $355^{2}, 504$

Colomb, Hector 483 
Colombo, Eduardo $405^{154}, 504$

Combes, Claudette $355^{2}, 504$

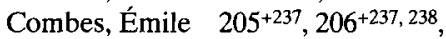
$207+239,240$

Comment, Bernard $435^{313}, 504$

Condette, Jean-François $321^{94}, 504$

Condorcet Marie Jean Antoine Nicolas Caritat, Marquis von 53

Constans, Jean 68

Cooper, Barbara T. $301^{36}, 356^{5}, 504,520$

Corbin, Alain $92^{36}, 117^{127}, 356^{4}, 454^{321}$, 503, 504

Corday, Charlotte 41

Cosgrove, Denis $452^{311}, 504$

Courbet, Gustave $102,104^{82}, 105^{+87}, 106$

Courtet, Emile 479

Couturier, Edouard 479

Couturier, Louis $290^{14}, 319^{92}, 325$, $334^{+136,138}, 376,387^{86}, 479,491$

Crafton, Donald $82^{82}, 259^{111}, 504$

Craïb 478

Crampe-Casnabet, Michèle $32^{56}, 504$

Crary, Jonathan $14^{63}, 298^{30}, 335^{139}, 140$, $336^{144}, 377^{62}, 453^{313}, 455^{324}, 504$

Créhange, André $364^{32}, 365^{37}, 493$

Croilo 478

Cruishank, Isaac $192^{183}$

Cubitt, Geoffrey $185^{144}, 504$

Cueto-Asín, Elena $382^{72}, 429^{228}, 504$

Dalou, Aimé-Jules $104^{+82}, 235^{37}$, $345^{+170,171}, 344^{+294}, 479$

Daniel, Ute $4^{16}, 504$

Dardel, Aline $113^{116}, 339^{152}, 343^{163}$, $353^{193}, 400^{133}, 137405^{155}, 406^{156,159}$, $407^{162,163}, 408^{166}, 441^{284}, 456^{331}, 500,504$

Darjou, Alfred 94, 99, 497, 487

Daumier, Honoré $11,22^{93}, 55^{+96}, 57$, $58^{+111}, 59^{+121}, 60^{+121}, 61,64^{6}, 70^{36,37}$, $71^{+38,39,40}, 72^{+43}, 76^{53}, 77^{56}, 83^{84}, 85^{+5}, 86$, $88,112^{113}, 123^{147}, 1251^{53}, 149,150^{25}, 157$, $163,168,232,265^{133}, 324^{98}, 346^{177}, 347^{180}$, $357^{13}, 381,388^{89}, 477,479,487$

David, Jacques-Louis 41

Dayle 477

Debat-Ponson, Édouard-Bernard 479

Debierre, Charles 209

Debuchy, Victor $97^{46}, 504$

Defaux, Gérard $30^{43}, 495$

Defrance, Eugène $178^{122}, 179^{125}, 359^{22}$, $360,375^{56}, 382^{72}, 493$

Delacroix, Eugène $132^{+182}, 149^{21}, 234$

De La Motte, Dean $129^{175}, 504,513,517$
Delannoy, Aristide $\quad 72,282,283,479,489$

Delattre, Simone $99^{63}, 100^{64}, 101^{74}$, $356^{4,7,8}, 357^{12}, 377^{61}, 384^{78}, 387^{87,88}$, $388^{+90,91}, 389^{94}, 392^{106}, 504$

Deligne, Alain $338^{149}, 504,511$

Delon, Michel $4^{19}, 32^{56}, 34^{6}, 44^{38}, 505,522$

Delord, Taxile 59

Delort, Robert $440^{279}, 505$

Delporte, Christian $8^{34}, 63^{1}, 65^{11,13}$, $77^{57,59}, 81^{79}, 287^{4}, 497,505$

Delteil, Loys $55^{96}, 479,487,498$

Demandt, Alexander $29^{31}, 46^{49}, 505$

Demisch, Heinz $120^{140}, 446^{296}, 505$

Demoris, René $33^{65}, 503$

Denkel, Eugen $177^{115}, 505$

Deonna, Waldemar $50^{70}, 505$

Descartes, René $27^{+21}, 48,324,335^{139}$, 472, 493

Désile, Patrick $11^{48}, 253^{99}, 357^{11}, 455^{326}$, $459^{338}, 473^{14}, 505$

Desloges $156^{46}$

Desperret, Auguste $56,57^{107}$

Dewitz, Bodo von $337^{148}, 505,520$

Diderot, Denis $31^{+49}, 33^{65}, 335^{139}, 431,493$

Diogenes $135,137^{+194}, 266^{137}, 387^{+89}, 465^{4}$

Disdéri, André $112^{113}$

Dittmar, Peter $80^{74}, 270^{151}, 290^{14}, 302^{41}$, $304^{47}, 385^{82}, 405^{155}, 429^{230}, 444^{292}, 449^{302}$, $457^{332,}, 334,459^{336}, 505$

Dixmier, Elisabeth $77^{55}, 505$

Dixmier,Michel $77^{55}, 160^{61}, 163^{73}, 184^{193}$, $188^{163}, 192^{181}, 198^{204}, 353^{54}, 505$

Doizy, Guillaume $184^{139}, 505$

Dölger, Franz Joseph 3911, 505

Donahue, Thomas Francis $11^{46}, 505$

Donaldson-Evans, Mary $301^{36}, 356^{5}, 504$, 520

Don Quichote $\quad 60^{124}, 147$

Don Zed 485

Douat, Albert 478

Doucet, Hervé $436^{264}, 505$

Doumergue, Gaston $203^{225}$

Draner $96,97,135,137,15748,51,167,174$, $175,177,178^{122}, 180^{+131}, 181,186,194$, $250^{48}, 241^{54}, 256,364,381,388^{89}, 440$, $452,477,479,484,487,488,492,496$

Dresch, Jutta $442^{289}, 505$

Dreyfus, Alfred $289^{+7,9}, 290^{+11}, 293^{+17}$, $294,295,301^{37}, 302,304,305,306,307$, $309,311,312^{71,72}, 314,316,319,321$, $321^{92}, 326^{108}, 341^{156}, 489$

Drost, Wolfgang $211^{254}, 229^{15}, 441^{282}, 505$, 511 
Drouin, Michel $287^{1,2}, 288^{4}, 292^{16}, 304^{44}$, $311^{66}, 312^{68,71}, 316^{78,80,82}, 317^{85}, 502$, $506,507,515,516,517,518$

Drummond, Thomas $177^{115,116}, 505$

Drumont, Édouard $345,436+261,493,497$

Duby, Georges $169^{95}, 389^{98}, 500,507,508$, 516

Du Camp, Maxime $91^{68}, 357^{11}, 493$

Ducatel, Paul $21,87^{8}, 89^{17,20}, 124^{148}$, $132^{181}, 149^{23}, 231^{22}, 242^{57}, 387^{89}, 487,498$

Duccini, Hélène $64^{5}, 507$

Duclert, Vincent $288^{6}, 506$

Du Fayl 494

Dumas, Alexandre 494

Du Moncel, Théodore $176^{109}, 493$

Duplessis, Joseph-Siffrède 479

Duprat, Annie $8,38^{5}, 40^{12}, 52^{80}, 506$

Dupuy, Aimé $63^{3}, 506$

Dupuy, Charles 332

Dürer, Albrecht $212^{261}$

Dutiers, Émile 362

Duveyrier, Charles 432

Echard, William E. $229^{15}, 232^{23}, 506$

Eckert Boyer, Patricia $79^{69}, 418^{204}, 503$, 506

Edison, Thomas Alva $161^{63}, 176^{+109}$, $241^{+52}, 360^{25}$

Edward VII. 457

Edward, George $289^{7}, 480$

Egbert, Donald Drew $396^{112}, 407^{163}, 506$

Ehrly, Idan $403^{+146}, 404^{148}, 434,435^{254}$, 436

Eliade, Mircea $39^{11}, 444^{294}, 506$

Elwitt, Sanford $162^{66}, 166^{82}, 506$

Énault, Louis $105^{+86}, 128^{166}, 164^{+79}, 502$

Endell, August $362^{26}, 384^{79}, 493$

English, Donald E. $112^{113}, 337^{148}, 506$

Es/d'Es (auch: Valdès/Val d'Es) 485

Escobar y Mendoza, Antonio de $197^{202}$

Esterhazy, Marie Charles Ferdinand

Walsin $302,311,314,316,328,328^{120}$, $334,351^{188}$

Evenson, Norma $398^{98}, 391^{102}, 506$

Ewald, François $166^{82}, 167^{85}, 506$

Faggin, Giuseppe $3^{10}, 506$

Fau, Fernand $269^{147}, 270^{148}, 375,480$

Faure, Félix $289^{8}, 327,328^{120}, 400$

Faure, Sébastien $289,290^{11,12}, 397^{118}$, $400^{134}, 497$

Favre, Jules 89

Felber, Ulrike $238^{44}, 506$
Ferry, Jules $\quad 68,161,174,198,200^{209}, 212$, $265,265^{131}, 267,269,309,357^{179}$

Festa, Nicola 495

De Feure, Georges 480

Fevre, D. 480

Feyel, Gilles $87^{59}, 506$

Fidus $454^{323}, 460^{340}$

Figuier, Louis $177^{116}, 256^{106}, 371^{49}, 493$

Filozel 482

Fischer, Hubertus $9^{+36}, 12^{49}, 38^{7}, 39^{10}$, $44^{37}, 51^{75,76}, 52^{78,80,83}, 53^{84,85,86,90}, 54^{94}$, $55^{97}, 57^{107,108}, 59^{111,114}, 59^{120}, 60^{124}, 99^{61}$, $141^{1.2}, 143^{4.5 .6}, 149^{21}, 153^{35}, 154^{+42}, 168^{88}$, $192^{183}, 200^{215}, 311^{65}, 312^{69,72}, 408,506$

Fleckner, Uwe $11^{48}, 117^{125}, 506,509,513$

Fleming, Marie $437^{268}, 506$

Floquet, Charles $161^{+64}$

Fô 480

La Fontaine, Jean de $147^{+18}, 149^{+20}, 494$

Fontane, Charles $65^{14,15}, 66^{18}, 506$

For 480

Forain, Jean-Louis (auch: Jean) $78^{62}, 82^{82}$, $233^{29}, 312^{71}, 340,414,415,480,491,497$

Forgione, Nancy $382^{72}, 506$

Forth, Christopher E. $295^{26}, 298^{28.29}$, $302^{39}, 304^{46}, 307^{57}, 506$

Foucault, Michel $4^{16}, 338^{150}, 461^{1}, 474^{+16}$, 506.

Fourier, Charles 331,332

Fournel, Victor $104^{+84}, 105^{85}, 106^{98}, 493$

Fournier, Éric $105^{86}, 507$

Fox, Robert $176^{109}, 507$

Franklin, Benjamin $44^{+38}, 48^{64}, 53,238$

Frey, Andrea $377^{62}, 507$

Friedrich, Caspar David $454^{323}$

Fritscher, Bernhard $46^{55}, 507$

Fuchs, Eduard $71,72^{41}, 78^{62}, 79^{67}, 80^{75}$, $132^{184}, 494$

Fugier, Anne-Marie $\quad 169^{95}, 507$

Furet, François $37^{2}, 507,516$

Gache-Patin, Sylvie $\quad 433^{244,245,247}, 507$

Gage, Jean $19^{85}, 507$

Gaillard, Jeanne $90^{26}, 91^{28.33}, 117^{126}, 507$

Gaillard, Marc $242^{58}, 357^{9}, 358^{15}, 507$

Gallarmand 480

Galliffet, General Gaston, Marquis de $118^{+38}, 119,120^{139}, 487$

Gallo, Armand $378,480,491$

Gallo, Max $188^{163}, 507$

Gambetta, Léon $68,135,166^{+84}, 167$, $184^{+142}, 198,212,218^{+282}, 220,266,494$

Gardes, Jean-Claude $\quad 64^{5}, 507,511$ 
Garnert, Jan $169^{95}, 338^{151}, 507$

Garrigues, Jean $64^{5,8}, 116^{122}, 141^{3}, 156^{43}$, $159^{59}, 242^{62}, 312^{71}, 507$

Gautier, Théophile $91^{+31}, 494$

Gavarni 494

Gavroche 108

Gay, Jean $100^{67}, 507$

Genette, Gérard 1983, 507

George-Edward 480

Gérault-Richard $121^{142}, 444^{292}$

Gersmann, Gudrun $242^{59}, 512$

Gervereau, Laurent $21^{92}, 79^{67}, 287^{2,4}$, $337^{148}, 348^{184}, 4362^{65}, 499,507$

Gibet $388^{91}, 478$

Giersch, Ulrich $454^{320}, 455^{324}, 507$

Gilbert-Martin, Charles $64^{+9}, 159,163$, $191,192,231,232,257,387^{89}, 390,496$

Gill, André $\quad 60^{124}, 65^{+14}, 66^{+16,17,18,19}$, $77^{56,59}, 82,150,151,152,154,155,158$, $205^{232}, 226,227,228,231^{21}, 234,235^{+34}$, $242,257,262^{114}, 444^{293}, 480,482,488$, 489,497

Gill, Susan $1201^{41}, 270^{151}, 355^{179}, 385^{81}$, $405^{155}, 503$

Girard, Eugène $140^{178}, 480$

Girard, Jean-Claude $200^{213}, 508$

Girardet, Raoul $185^{146}, 508$

Goblet, René $198,200^{+211}$

Goldstein, Robert J. $63^{2}, 64^{5,7,9}, 66^{18}$, $67^{22}, 69^{32}, 70^{35}, 72^{+43,44,45}, 73^{47,48,49}, 508$

Golinski, Hans-Günther $382^{72}, 508$

Gombrich, Ernst $37^{1}, 508$

Gosset de Guines, André 480

Gottlob, Fernand 205, 206, 385, 480, 488

Goulemot, Jean-Marie $191^{175}, 508$

Goussé, Henri 416,480

Grad, Bonnie L. $389^{92}, 433^{241}, 508$

Grand-Carteret, John $184^{139}, 197^{200}$, $206^{237}, 287^{4}, 470^{10}, 494$

Grandjouan, Jules-Félix $\quad 9^{38}, 77^{55}, 80^{+76}$, $186^{152}, 208^{246}, 340,348^{+184}, 349,365,366$, $367,375,383,384,401^{140}, 410^{170}, 431$, $480,490,491$

Grandville, Jean-Ignace Gérard $53^{85}$,

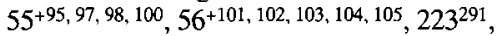
$232^{+27}, 480$

Grave, Jean $343^{163}, 399,400,408^{168}, 437$, 497

Gravelle, Émile $\quad 435,436^{267}, 498$

Gravelot, H.-F. $\quad 102^{75}, 140,495$

Green, Nicholas $433^{242,243}, 508$

Grégori, Louis A. 321

Grenèche, Louis 496
Grenet, Micheline $\quad 4^{18}, 4965,508$

Grévy Jérôme $184^{182}, 508$

Grévy, Jules $159,184^{142}, 265^{+130}, 267,269$, $309,388^{91}$

Griewank, Karl $40^{15}, 41^{17}, 49^{66}, 508$

Griffiths, Richard $288^{5}, 299^{32}, 301^{36}, 508$

Grippa, A. 220, 221, 480, 488

Grünewald, Dieter $83^{84}, 508$

Guédy, P. 496

Guerrand, Roger-Henri $389^{98}, 432^{240}, 508$

Guesde, Jules $273^{157}, 289^{9}, 410^{+171}, 452$, 498

Guillaumin, Edouard 484

Gullickson, Gay L. $\quad 13^{51}, 129^{175}, 130^{176,178}$, 179, $180,132^{182}, 508$

Gumery, Adolphe $305,306,307,480$

Habert, Eugène $75,480,487$

Hadol, Paul 167, 185, 440, 481, 488, 492

Haenen (auch: Haenens), Frédéric de $293^{17}, 481$

Haine, Scott W. $391^{103}, 508$

Halsberghe, Gaston $\mathrm{H}$. $\quad 39^{11}, 508$

Hamon, Léo $166^{82}, 184^{140}, 506,514$

Harten, Elke $43^{34}, 50^{70}, 508$

Harten, Hans-Christian $43^{34}, 50^{70}, 508$

Harvey, David $128^{164}, 189^{164,170,171}, 508$

Haskell, Francis $48^{63}, 70^{37}, 508$

Hauk, Gerhard $460^{340}, 508$

Haussmann, Georges-Eugène Baron $112^{113}, 356^{+5}, 357^{9}, 373^{52}$

Hautecour, Louis $40^{12}, 508$

Havelange, Carl $324^{101}, 508$

Hawkins, Louis Welden $327^{+116}, 481$

Hazareesingh, Sudhir $226^{10}, 509$

Heidbrinck, Oswald $248,362,363,371$, $384,395^{110}, 401,402,481,490,491$

Heine, Thomas Theodor $348^{181}$

Heinrich IV. 432

Hellé $420^{+209}, 421,481,491$

Heller, Hartmut $\quad 471^{11}, 509$

Heller, Steven $79^{69}, 302^{40}, 407^{163}, 520$

Héma 477

Hempel, Johannes $2^{5}, 509$

Hénault, Jules $171,172,185^{148}, 343^{+163}$, 481,488

Henri IV, s. Heinrich IV.

Henriot $140,332^{+131}, 481$

Herbert, Eugenia W. $396^{112}, 509$

Herding, Klaus $8^{+31}, 37^{1,4}, 38^{5.7,8}, 39^{10}$ $40^{13,16}, 41^{22,23}, 44^{37}, 45^{46}, 46^{49,51}, 47^{56}$ $60^{70}, 98^{56}, 105^{87}, 160^{60}, 388^{89}, 440^{278}, 509$

Herkules $160^{60}$ 
Hermann, F. $287^{4}, 351^{188}, 509$

Hermann-Paul $71^{14}, 275,276^{171}, 290^{14}$, $304^{44}, 317^{85}, 327^{+114,118}, 330^{+124,125}, 332$, $341^{156}, 351^{188}, 386,481,493,496$

Hermann, René-Georges Paul 481

Héron de Villefosse, Angélique $246^{76}$, 509

Heymann, S. $\quad 66^{19}, 497$

Hick, Ulrike $61^{127}, 473^{15}, 509$

Hiekisch-Picard, Sepp $77^{55}, 79^{68}, 509$

Hillaret, Jacques $176^{107}, 509$

Hirdina, Karin $473^{15}, 509$

Hodeir, Catherine $186^{156}, 191^{173}, 509$

Hofmann, Werner $48^{62}, 509$

Hohl 212 261, 509

Holländer, Hans $457^{333}, 509$

Hölzel, Volkmar $99^{58}, 509$

Hoormann, Anne $473^{15}, 509$

Hope $389^{95}, 390,481,491$

Höppener, Hugo, s. Fidus

House, John $117^{125}, 483,509$

Houssaye, Arsène 494

Howlett, Marc-Vincent $26^{12}, 510$

Hugo, Victor $57^{56}, 85,86^{6}, 92^{+37}, 97^{46}$, $108^{109}, 126^{+159}, 149,151^{+31}, 166^{+83}, 167$, $168,191^{+178.179}, 192,216,244^{+67}, 353^{192}$, $355^{2}, 431,434^{+252}, 494,498$

Husson, Jules François Félix, s. Champfleury

Hüttinger, Eduard $287^{4}, 509$

Hutton, John G. $114^{119}, 273^{160}, 274^{164}$, $404^{151}, 407^{163}, 438^{276}, 446^{295}, 448^{298}$, $456^{328}, 457^{335}, 510$

Hutton, Patrick H. $89^{17}, 118^{138}, 153^{33}$, $159^{58}, 161^{64}, 200^{209,211}, 203^{222}, 205^{237}$, $207^{241}, 259^{113}, 263^{120}, 265^{130 .}{ }^{131}, 289^{9}$, $312^{71}, 326^{110}, 345^{170}, 388^{91}, 420^{208}, 422^{212}$, $429^{231}, 510$

Huysmans, Joris Karl $272^{156}, 494$

Ibels, Henri-Gabriel $1^{+1}, 78^{65}, 80^{+73}, 82^{82}$, $195^{+192}, 299,300,301,302,316,317,319$, $325,327,328,330,330^{127}, 331,334^{134,135}$, $341,343,345,346^{175}, 352,481,488,490$, 496,497

Ignatius von Loyola 197

Ihl, Olivier $191^{175}, 254^{101}, 264^{123}, 510$

Imdahl, Max 495

Im Hof, Ulrich $32^{56}, 510$

Iribe, Paul 497

Isaacson, Ester $377^{62}, 456^{328}$

Isaacson, Joel $79^{70}, 510$

Isoré, Luis 191, 481
Jablochkoff, Pawel Nikolajewitsch $176^{+111}, 177,178^{122}, 179^{125}, 180^{131}, 194^{189}$, $229,231,241^{52}$

Jacob, Margaret C. $\quad 48^{64}, 510$

Jacquemet, Gérard $365^{35}, 510$

Jacquet 482

Jäger, Hans-Wolf $38^{6}, 42^{27}, 44^{38}, 45^{45}$, $46^{50}, 510$

Jankowski, Ch.-B. $\quad 423,424,481,491$

Janowski, Bernd $2^{7}, 510$

Jansen, Michael $457^{333}, 509$

Jaurès, Jean $127,200^{+212}, 289^{+9,10}, 304^{+48}$, $306,312^{71}, 313^{72}, 411^{179}, 423,426^{+220}$, $471,494,498$

Jauss, H. R. $241^{54}, 245^{70}, 495,521$

Jeanne d'Arc $212^{259}, 218$

Jehannet 353,481

Jeziorkowski, Klaus $460^{340}, 510$

Job 269,481

Joest, Thomas von $225^{3.5}, 226^{8}, 233^{31}, 510$

Johnson, Martin P. $\quad 287^{1}, 328^{120}, 510$

Joly, Agnès $40^{12}, 510$

Jonard, Robert $42^{27}, 510$

Jonas, Raymond A. $189^{167}$

Jones, Philippe, s. auch Roberts-Jones, Philippe $21^{89}, 65^{14}, 66^{16}, 70^{+33.34}, 76^{+53}$, 510,518

Jordan, Stefan $19^{85}, 29^{31}, 505,515$

Jossot, Gustave-Henri $389^{96}, 481$

Josua $87^{8}$

Jouve, Paul 251, 482

Jover, Manuel $80^{72}, 506$

Jullien, Vincent $26^{12}, 501,504,510$

Jung, Ruth 510

Jupiter $44^{38}, 45^{+39.40}, 423$

Justus, B. $26^{16}, 510$

Kab $82,205^{+23}, 206,325^{106}, 327,329$, $470^{10}, 482,483,490$

Kaenel, Philippe $236^{43}, 238^{44}, 338^{151}, 511$

Kalifa, Dominique $373^{54}, 375^{55.58}, 511$

Kaluza, Zénon $26^{12}, 511$

Kampmeyer-Käding, Margret $90^{26}, 511$

Kantorowicz, Ernst H. $40^{12}, 511$

Kape 482

Karl X. $\quad 57^{107}, 224$

Kauffmann, Peter Paul 147, 148, 149, 482 , 488

Kaulbach, Hans-Martin $\quad 400^{138}, 511$

Kedward, H.R. $\quad 288^{+6}, 511$

Keller, Dominik $40^{12}, 511$

Kelly, David $355^{2}, 504$

Kemp, Wolfgang $17^{75}, 264^{+126}, 441^{282}, 511$ 
Kerbs, Diethard $436^{264}, 512$

Kern, Stephen $11^{47}, 511$

Kessler, Marni $113^{115}, 511$

Kirchmann, Kay $11^{46}, 511$

Klauser, Theodor $19^{86}, 507$

Kleeblatt, Norman L. $\quad 287^{2}, 287^{4}, 304^{45}$, $306^{54}, 307^{60}, 316^{76}, 321^{97}, 327^{118}, 328^{122}$, $338^{149}, 339^{152}, 353^{194}, 502,503,511$

Kleinspehn, Thomas $335^{139}, 511$

Klenck, Paul $22^{94}, 87^{8}, 130^{178}, 157^{+49}$, 482

Koch, Hal $197^{202}, 511$

Koch, Ursula E. $8^{+33}, 55^{95}, 58^{115,117}, 68^{30}$, $76^{+52,53}, 87^{7}, 511$

Koerber, Rolf $436^{264}, 512$

Kohle, Hubertus $242^{59,61}, 245^{68,72}, 247^{81}$, $248^{84}, 264^{126}, 512$

Kohler, Georg $\quad 40^{12}, 232^{25}, 511,512$

Köhler, Hubert $444^{294}, 512$

König, Mareike $388^{90}, 512$

Konstantin 248

Koopmann, Helmut $42^{27}, 512$

Kopernikus, Nikolaus $4^{18}, 40,49^{67}$

Koschorke, Albrecht $453^{315}, 454^{323}, 512$

Koselleck, Reinhart $4^{+17}, 5^{20}, 17^{78}, 30^{39}$, $31^{46}, 42^{28}, 43^{33}, 242^{59}, 512,521$

Koslofsky, Craig $97^{50}, 512$

Krause, Gerhard 25,501

Krauter, Anne $473^{15}, 512$

Kretschmer, Winfried $225^{3}, 242^{58}, 244^{64}$, $248^{87}, 253^{99}, 512$

Kristeva, Julia $18^{80}, 461^{1}, 512$

Kropotkine, Pierre $438^{275}, 494$

Krumeich, Gerd $211^{255,256}, 212^{259}, 218$, $2182^{79}, 224^{296}, 512$

Kupka, František $193^{+185}, 240^{50}, 348^{151}$, $339^{+153}, 437^{+268}, 460^{340}, 482,488$

Kurz, Gerhard $7^{28}, 512$

Laclôtre, André 481

Ladreyt, Eugène 157,482

Lafosse, Georges 480

Lagarde, Maurice 497

Lagrée, Michel $20^{87}, 129^{171}, 133^{189}, 194^{191}$, 512

Lalaux, Jean-Bernard $\quad 184^{139}, 505$

Lalouette, Jacqueline $160^{61}, 163^{73,75,77}$, $184^{139}, 185^{146}, 188^{160,162,163}, 189^{164,166,168}$, $192^{181}, 198^{204}, 213^{265}, 505,512$

Lambert, Gisèle $77^{55}, 513$

Lami, Eugène 494

L'Aminot, Tanguy $436^{262}, 437^{267}, 513$

Lamouche, Eugène $\quad 263^{+121}, 388^{89}, 482$
Langemeyer, Gerhard $77^{55}, 79^{68}, 97^{45}$, $346^{177}, 347^{180}, 348^{181}, 382^{72}, 385^{82 / 83}, 508$, 509,513

Langlois, Claude $8,38^{9}, 43^{35}, 513$

Lardy, Jacques $411^{177,}{ }^{179}, 412^{183}, 416^{195}$, 494

Larkin, Maurice $216^{268}, 340^{154}, 513$

Larmand 480

Lasky, Melvin J. $47^{57}, 396^{114}, 462^{2}, 513$

Latocha, Rita $436^{264}, 455^{323}, 460^{340}, 502$, 522,524

Launay 482

Lavoisier, Antoine Laurent de 53

Lay, Howard G. $399^{129}, 400^{137}, 442^{290}$, 513

Lazare, Bernard $292^{+16}, 311,494$

Lazare, Louis $91^{+34}, 191,357^{12}, 494$

Léandre, Charles $203^{225}, 208,209,290^{14}$, $326^{+108}, 412,413,414^{188}, 482,491$

Lebasque, Henri $385,441^{284}, 482$

Le Bon, Gustave $298^{+29,30}, 302^{42}, 494$

Lebovici, Élisabeth $232^{26}, 233^{30}, 502$

Lecocq, Charles $154^{38}$

Lecouvey, Hélène $404^{151}, 513$

Lefebvre, Jules $236^{43}, 309^{+62}, 310,482$, 490

Lefman $109,482,487$

Le Grand 482

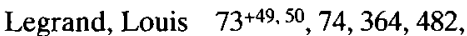
487

Legrand, Marc $384^{+79}, 395$

Lehning, James R. $262^{117}, 264^{123}, 267^{141}$, $269^{145}, 513$

Leith, James A. $\quad 44^{38}, 88^{12}, 89^{21}, 90^{24}$, $107^{101}, 116^{123}, 126^{159}, 130^{178}, 191^{175}, 513$

Lek 482

Lemaire $51^{77}, 482$

Le Men, Ségolène $11^{+48}, 61^{126,127}, 88^{16}$, $118^{133}, 124^{149}, 150^{24,25}, 389^{96}, 499,513$, 521

Lemer, Julien $229^{15}, 494$

Lemot, A. $1^{+2}, 82^{+8 t}, 124,198,199$ $201^{+218}, 202,209,210,216,218^{276,278}$, $219,224^{297}, 347^{179}, 482,483,488$

Le Petit (auch: Lepetit), Alfred $72^{45}, 123$, $132^{182}, 141,142,143^{6}, 144,146,151,154$, $158,161,181,182,192,209^{250}, 218^{282}$, $254,255,313^{72}, 368,482,488,489,497$

Lerory 483

Lethève, Jacques $63^{2}, 72^{45}, 73^{47}, 77^{56}$, $81^{79}, 85^{3}, 184^{141}, 198^{204}, 205^{235}, 311^{65}, 513$

Levin, Miriam R. $242^{59}, 245^{69}, 248^{82}$, $250^{88,92}, 251^{95}, 274^{161}, 513$ 
Lévy, Alphonse $385,477,483,484$

Lévy, Arthur 497

Lilio $138,482,483,488$

Link, Jürgen $4^{15,16}, 7^{28}, 10^{+44}, 37^{3}, 513$, 516

Linse, Ulrich $440^{279}, 441^{281}, 513$

Lippincott, Louise $12^{49}, 61^{127}, 100^{69}$, $169^{93}, 176^{112}, 192^{182}, 183,194^{190}, 229^{15}$ $236^{38}, 240^{49}, 241^{52}, 245^{71}, 250^{91}, 272^{156}$, $356^{7}, 357^{9}, 359^{23}, 367^{41}, 392^{107}, 473^{14}, 501$

Lix, Frédéric 130,483

Looft-Gaude, Ulrike 139200,513

Loubet, Émile $207^{+241}, 345$

Louis XIV, s. Ludwig XIV.

Louis XVI, s. Ludwig XVI.

Louison 485

Louis-Philippe $56^{+103}, 57^{+107}, 71,88$, $156^{+46}, 224,232,259^{113}, 265^{133}, 388^{89}$

Löwith, K. $\quad 10^{+42}, 514$

Loyer, François $188^{64}, 357^{9}, 373^{51}, 514$

Loyrette, Henri $\quad 245^{69}, 248^{83}, 514$

Luc 483

Luce, Maximilien $80,82,290^{14}, 305,306$, $339,389^{129}, 400^{+137}, 404^{151}, 406,438^{276}$, 483

Luckmann, Thomas $16^{+69}, 501$

Ludwig XIV. $\quad 49^{+67}, 97,212,494$

Ludwig XVI. $38^{+5}, 125^{154}, 150^{25}, 224$

Ludwig XVIII. 52

Luh, Peter $\quad 68^{27}, 185^{149}, 514$

Luhmann, Niklas $16^{+71}, 514$

Lunel, Ferdinand 483

Luque, Manuel $130^{181}, 161^{+63}, 483$

Lüsebrink, Hans-Jürgen $6^{+22}, 32^{56}, 33^{61}$, $40^{12}, 514$

Lüthy, Hans A. $287^{4}, 509$

Lys, Georges de $448^{298}$

Maag, Georg $241^{54}, 514$

Mabire, Christophe $248^{87}, 250^{92}, 514$

Machaux, Adrien 477

Mac-Mahon, Marie Edmé Patrice Maurice, Comte de $67,116^{122}, 117^{125}, 141,149$, $153,156^{43}, 159,226,309$

Magne, Jacqueline $82^{82}, 514$

Mahlmann, Th. $32^{56}, 519$

Maigrot, Henri 481

Maillard, Firmin $63^{3}, 89^{21}, 494$

Maillet, Joseph oder Jean-Joseph 483

Maitron, Jean $343^{162}, 396^{112}, 398^{126}$, $399^{127 .} 130,400^{134}, 410^{171,172}, 435^{257}, 514$

Malachias 209

Malmede, Hans $2^{8}, 3^{10}, 514$
Mandell, Richard $248^{87}, 514$

Manet, Édouard $100^{69}$

Manevy, Raymond $63^{1}, 514$

Mann, Heinz Herbert $185^{146}, 514$

Marat, Jean-Paul $41,51^{75}, 107,375,388^{90}$

Marcellus, M. 496

Maréchal, Henri $178^{121}, 183^{136,137}, 358^{15}$, $359^{17,21,22}, 360^{+25}, 361,362^{27}, 368^{43}$, $373^{52}, 490,494$

Maréchal, Sylvain $39^{10}, 47^{56}$

Mareske, Christina $245^{71}, 245^{77}, 248^{87}$, $250^{89}, 514$

Marianne $8,132^{184}, 156^{43}, 203^{225}, 207$, $224,233,236,251,253,259,267,270^{148}$, $284,285,314,315,316,317^{84}, 346^{179}, 431$, 490

Mariaux, Pierre-Alain $406^{155}, 514$

Marie-Antoinette $124,125^{154}, 287^{86}$

Markovits, Francine $33^{64}, 514$

Martel, Tancrède $362^{29}, 497$

Martial $146^{13}, 442,445,446,449,483,492$

Martin, Marc $63^{1}, 77^{57}, 514$

Marville, Charles $357^{9}, 522$

Marx, Karl 431, 452

Matz, Jutta $139^{200}, 192^{183}, 513,514,523$

Maurin, Charles $399^{126}, 483$

Mayeur, Jean-Marie $117^{127}, 184^{140}, 208^{242}$. $245,209^{248}, 503,514$

McDonough, Tom $113^{115}, 511$

Mehl, Heinrich $139^{200}, 192^{183}, 513,514$, 523

Méline, Jules $316^{+78}$

Melot, Michel $71^{38,40}, 514$

Ménager, Daniel $30^{38}, 514$

Mensching, Gustav $2^{5}, 514$

Mercier, Auguste 334

Mercier, Louis-Sébastien 33, 3466, 494

Méria, Adrien 477

Merle, Gabriel $205^{237}, 206^{238}, 514$

Merriman, John M. $91^{33}, 113^{113}, 514$

Métivet, Lucien 418,483

Meyer, Henri $293^{17}, 294,309^{+62}, 310,473$, 489,490

Michaud, Stéphane $163^{77}, 513$

Michel, Louise $130^{+181}, 269^{+144}$

Michel, O. $2^{12}, 515$

Millaud, Polydor 65

Mille, E. 482

Millon, Henry A. $81^{77}, 520$

Milner, John $104^{82}, 105^{87}, 117^{127}, 515$

Milza, Pierre $207^{239}, 357^{10}, 501,516$

Mirbeau, Octave $302^{42}, 496$

Mirande, Henri $\quad 274,386,483$ 
Mobb $87^{8}, 483$

Molière 197

Mollenhauer, Daniel $\quad 160^{62}, 444^{292}, 515$

Mollier, Jean-Yves $63^{1}, 77^{58}, 163^{77}, 513$, 515

Moloch $69^{32}, 89^{17}, 208,242,243,244^{65}$, $247,251,256,258,259,261,263,282$, $456^{328}, 483,489$

Momus 483

Money, E. $71^{37}, 494$

Montalembert, Comte de $58^{+111}$

Montelli 485

Montorgueil, Georges $388^{92}, 494$

Moreau-Nélaton, Adolphe Étienne Auguste $359^{23}, 469^{9}, 483$

Morel-Retz, Pierre Gabriel Bernard 485

Morice, Léopold 235, 236

Morin, Edmond $238,239,241^{+55}, 246$, 483, 489

Morland, Valère $82,478,482,483$

Mortier, Arnold 497

Mortier, Roland $32^{56}, 33^{58,61}, 41^{24}, 324^{101}$, 497, 508, 515, 520

Möseneder, Karl $39^{12}, 158^{51}, 515$

Mosser, Monique $46^{53}, 50^{69}, 515$

Müller-Hofstede, Justus $332^{132}, 515$

Munholland, John Kim $270^{152}, 278^{175}$, 515

Murdorfer, Anthony $185^{145}, 494$

Musset, Paul de 494

Napoleon I. $51,52^{+80}, 143,151^{+31}, 153^{35}$, $159,161,181,224,226^{10}, 232,259^{113}$

Napoleon III. (Louis-Napoléon) 58, 61 , $66^{18}, 71,87^{8}, 91,126^{159}, 150,154,158$, $174,224,226^{10}, 236$

Napoléon Eugène Louis Jean Joseph, prince impérial $259^{113}$

Nataf, André $\quad 435^{257}, 515$

Nathan, Michel $105^{87}, 133^{188}, 515$

Nathanson, frères 496

Necker, François 37

Néraudau, Jean-Pierre $40^{12}, 515$

Nero 420

Neumann-Spallert, L. X. $\quad 177^{114}, 494$

Newton, Isaac $33,48^{+63,64}, 49$

Nicoullaud, Charles 497

Nochlin, Linda $81^{77}, 520$

Noé, Amédée, Comte de, s. Cham

Noiray, Jacques 495

Nolte, Paul $19^{85}, 515$

Nora, Pierre $188^{164}, 191^{175}, 245^{69}, 264^{125}$, $499,501,508,514,515$
Nord, Philip $301^{38}, 515$

Nox 485

Nye, David E. $247^{79}, 515$

Oberlé, Gérard $38^{5}, 47^{60}, 515$

Oechslin, Werner $473^{15}, 515$

Oettermann, Stephan $453^{313,314}, 515$

Ogé, Eugène $\quad 80^{76}, 188,189,484$

Ohly, Friedrich $29^{33}, 515$

Ola, D. $222^{287,288,289}, 288^{7}, 484$

Olano, David 484

Oriol, Philippe $292^{16}, 515$

Ory, Pascal $65^{11}, 242^{58}, 357^{10}, 505,515$

Osgood, Samuel M. $141^{1}, 259^{113}, 516$

Osietzki, Maria $240^{+51}, 516$

Osterwalder, Marcus 477, 516

O'Toole, J.-H. $\quad 80^{73}, 516$

Ottomeyer, Hans $440^{278}, 509$

Oudry, Léopold $360^{+25}, 361,490$

Oulman, Alfred 497

Ovid $40^{12}, 494$

Ozouf, Mona $37^{2}, 41^{20}, 43^{31,33}, 50^{72}$, $162^{66,67,70}, 163^{74}, 169^{92}, 173^{99}, 174^{105}$, $200^{210}, 256^{105} 273^{157}, 507,516$

Paf $194,477,479,484$

Pagès, Alain $312^{68}, 516$

Panofsky, Erwin $452^{312}, 516$

Papenheim, Martin $41^{18}, 381,516$

Parinet, Elisabeth $63^{1}, 516$

Parkhurst Ferguson, Priscilla $92^{37}, 434^{248}$, 516

Parmenides 25

Pascal, Blaise 31

Pataud, Émile $203,204,411,412,413$, $414^{188}, 416,417^{198,201}, 418,420^{+209}$, $422^{+211}, 423^{+218}, 424,471,488,491,495$

Paulson, Ronald $42^{27}, 516$

Pêcheux, Michel $4^{16}, 516$

Peirce, Charles S. $18^{79}$

Pelletan, Camille $388^{91}$

Pépin, Édouard $163,186,187,189,190$, $234,251,311,312^{72}, 313^{+72}, 314,328^{120}$, $334^{133}, 484,488,497$

Perrault, Charles $31^{44}, 495$

Perrig, Alexander $325^{103}, 516$

Perthuis, Bruno, de $312^{72}, 516$

Pessard $141^{2}$

Pessin, Alain $43^{31}, 90^{23}, 167^{86}, 396^{113}$, $404^{150}, 405^{154}, 406^{158}, 407^{164.165}, 442^{288}$, 504, 516

Petitjean, Honoré 481

Petit Pierre $82,484,485$ 
Petrarca, Francesco $30^{+40}, 495$

Petzina, Dieter $460^{340}, 508$

Pezilla, Mario $368,370,484,491$

Pfeiffer, Helmut $241^{54}, 245^{70}, 521$

Philipon, Charles $54,55^{100}, 57,99,496$

Philipp, Wolfgang $49^{68}, 516$

Philippe d'Orléans, Comte de Paris, (1838-1894) 259113

Philippe d'Orléans, Comte de Paris, (1869-1926) $259^{113}$

Philippe, Duc d'Orléans 38

Phô 480

Picinelli, Filippo $\quad 91^{75}, 495$

Picquart, Georges $302^{+39,43}, 304^{+44}$, $305^{+51}, 306^{+54}, 307^{+60}, 317^{85}, 327^{+118}$,

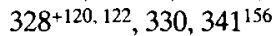

Pierrard, Pierre $128^{164}, 516$

Pierrot 485

Pif 481

Pilotell $88^{+11}, 92,114,126^{+158,160}, 484,496$

Pilotelle, Georges 484

Pissarro, Camille $79^{69}, 82,274^{162}, 356$, $377^{62}, 400,438^{276}, 448,456^{328}, 469^{8}, 484$, 495,496

Pissarro, Lucien $80,82,113^{+116}, 114$, $264^{127}, 399^{129}, 400^{+137}, 441,484$

Pius IX. $196^{198}$

Pius X. 1, 196, 209

Plagemann, Volker $65^{12}, 185^{149}, 520$

Plato $25^{+5.6}, 26,495$

Plessen, Marie-Louise von $8^{33}, 512,516$

Plitzner, Klaus $238^{44}, 240^{48}, 506,517,521$

Pohl, Klaus $460^{340}, 517$

Poidevin, Raymond $357^{10}, 516$

Poiré, Emmanuel 478

Poitevin 480

Polo, François 496

Poncet, Paul 408, 409, 484, 491

Poncet de Cluny, François $179^{127}, 360^{26}$, 495

Ponchon, Raoul 378

Ponet, Adolphe 496

Ponty, Janine $287^{2}, 517$

Pope, Alexander $48^{63}$

Potémont, Adolphe Martial 483

Poubelle, Eugène-René 389,391

Pouf 478

Pouget, Émile $399^{+129}, 400^{+134}, 410$, $417^{+198.201}, 420,442,495,497$

Poulin, Albert 497

Poutrin, Isabelle $171^{97}, 500$

Pradels, Octave 280

Preiss, Natalie $156^{46}, 493$
Prendergast, Christopher $120^{140}, 356^{3}$, $385^{80}, 432^{239}, 517$

Pressens, F. de $304^{44}, 495$

Prochasson, Christophe $14^{62}, 15^{64}, 21^{92}$, $78^{64}, 287^{2}, 301^{38}, 304^{44}, 307^{58}, 338^{148}$, $348^{184}, 410^{170}, 431^{234}, 499,507,517$

Prokasky, Judith $13^{52}, 517$

Prometheus $44^{38}, 85,344^{+169}$

Proudhon, Pierre-Joseph $396^{112}, 468^{+6}$, 495

Przyblyski, Jeannene M. $129^{175}, 399^{129}$, 504, 513, 517

Pucciarelli, Mimmo $405^{154}, 504$

Quatremère de Quincy, Antoine Chrisosthôme 41

Quesnay de Beaurepaire, Jules $317^{85}$

Quinsac, Paul-François $273^{160}, 484$

Rabelais, François $30^{43}, 495$

Rahner, Hugo $39^{11}, 517$

Raïeter, Gaston $284,285,484,489$

Ramnoux, Clémence $467^{5}, 517$

Ranc, Robert $76^{52}, 503$

Rapp, Friedrich $10^{41}, 31^{49}, 517$

Ratapoil 159, 171, 174, 180, 226, 231, 235 , 259, 345

Ratichon 477

Rault, Louis-Armand 304,484

Ravachol $398^{126}$

Rearick, Charles $264^{125}, 273^{157}, 158,383^{73}$, 517

Rebske, Ernst $\quad 97^{48}, 192^{183}, 356^{7}, 517$

Recchilongo, Benito $378^{62}, 517$

Reck, Hans Ulrich $46^{55}, 517$

Reclus, Élisée $\quad 437^{+268,273}, 460^{340}, 495$

Redon, Georges $314,315,484,490$

Reichardt, Rolf $3^{11}, 6^{+22.25}, 7^{+30}, 8,9^{+35.39}$, $37^{1,2.3 .4}, 38^{5}, 39^{10}, 40^{12}, 43^{33}, 44^{36}, 45^{46}$, $46^{49.51 .52}, 47^{56}, 50^{70}, 51^{73.75 .76 .77}, 52^{78.79 .83}$, $53^{84.85}, 54^{93.94}, 56^{105}, 57^{106.107 .108}, 58^{112}$, $76^{52}, 88^{15}, 98^{55}, 141^{1}, 160^{60}, 168^{89}$, $324^{98.101}, 463^{3}, 470^{10}, 509$

Reimbold, Ernst Thomas $2^{6}, 517$

Reinach, Joseph $312^{71}, 494$

Rémond, René $90^{22}, 162^{69}, 163^{75}, 184^{140}$, $186^{153}, 344^{165}, 518$

Renard, Jules 477,479

Renault, G. $448^{301}, 460^{341}$

Renaux 484

Reshef, Ouriel $85^{1}, 518$

Reulecke, Jürgen $436^{264}, 512$

Rey, Alain $90^{22}, 108^{107}, 518$ 
Reyem 483

Richard, A. 497

Richter, Michael $209^{251}, 518$

Ridley, F. F. $400^{135}, 410^{170}, 417^{197}, 418^{203}$, $422^{215}, 518$

Rieger, Dietmar $387^{89}, 518$

Riggs, Timothy A. $389^{92}, 433^{241}, 508$

Rigotti, Francesca $39^{11}, 325^{103}, 429^{229}, 518$

Rioux, Jean-Pierre $1^{3}, 14^{62}, 63^{1}, 242^{60}$, $245^{69}, 247^{80}, 515,517,518,520$

Ripa, Cesare $102^{75}, 495$

Ritter, Joachim 252, 509

Roberts-Jones, Philippe, s. auch Jones, Philippe $7^{29}, 64^{4,5}, 67^{21}, 510,518$

Robespierre, Maximilien 47, 124, 125+154, 126

Robichon, François $454^{319}, 518$

Robida, Albert $356^{17}, 484,496$

Roby 484

Roche, Daniel $\quad 98^{+53}, 518$

Rochefort, Henri $60^{124}, 200^{+213,216}, 244^{65}$, $262^{114}, 312^{+70,71}, 334$

Rodenberg, Julius $229^{15}, 495$

Roger, Alexandre $98^{54}, 153^{36}, 518$

Roger, Jacques $32^{56}, 33^{62}, 518$

Roland 484

Rollenhagen $42^{25}, 495$

Rollinat, Maurice $375^{56}, 495$

Römhild, Thomas $20^{86}, 518$

Rosenberg, Alfons $218^{276}, 406^{160}, 518$

Roslak, Robyn Sue $436^{263}, 438^{276}, 518$

Rosmer, Jan $357^{10}, 495$

Rothschild, Baron 137

Rouargue 494

Roubille, Auguste $406,407,484,496$

Rougerie, Jacques $\quad 90^{27}, 92^{39}, 93^{42}, 104^{83}$, 518

Roumieux, André $311^{66}, 518$

Rousseau, Jean-Jacques $43^{32}, 52,149^{21}$, $191^{175}, 225^{4}$

Rouvier, Maurice 208

Rudolph, Kurt $2^{9}, 518$

Rütten, Raimund $39^{10}, 43^{32}, 47^{56}, 59^{121}$, $60^{123}, 61^{125}, 66^{16}, 87^{7}, 99^{59}, 101^{71}, 105^{87}$, $112^{113}, 116^{123}, 130^{178}, 132^{184}, 159^{59}, 499$, $500,502,510,511,515,518,519$

Rysselberghe, Théodore von $328^{+122}, 484$

Sagave, Pierre-Paul $55^{95}, 58^{115,117}, 68^{30}$, 512

Saĩd $89,90,477,483,484$

Sainte-Croix, Camille de $278^{175}, 496$

Saint-Fourien 484
Saint-Martin, Catherine $22^{95}, 82^{80}, 158^{56}$, 486, 520

Saint-Patrice (= Baron Harden-Hickey) 497

Saint-Vidal, Francis de 247

Salé, Marie-Pierre $\quad 437^{268}, 519$

Salis, Rodolphe 496

Sánchez, Gonzalo J. $66^{19}, 67^{24}, 88^{11}$, $102^{78}, 104^{82}, 132^{186}, 168^{54}, 267^{141}, 519$

Sandherr, Jean 302

Sandy-Hook $388^{89}, 431,432,485,492$

Sanson, Rosemonde $234^{7}, 256^{104}, 264^{124}$, ${ }^{125}, 265^{128}, 273^{159}, 278^{174}, 279^{176,177}, 519$

Sauvage, Anne-Marie $80^{73}, 519$

Schabert, Tilo $43^{32}, 519$

Schaer, Roland $\quad 432^{240}, 504,507,519$

Schalk, Fritz $32^{56}, 519$

Schama, Simon $46^{53}, 433^{243}, 519$

Schapira, Marie-Claude $130^{178}, 132^{182}$, 519

Scheurer-Kestner, Auguste $311^{+66,67}$

Schiera, Pierangelo $39^{11}, 518$

Schivelbusch, Wolfgang $1^{4}, 11^{47}, 12^{49}, 85^{2}$, $97^{49}, 98^{51,54}, 99^{62}, 100^{64,65}, 118^{134}, 169^{93}$, $176^{111}, 241^{53}, 245^{72}, 246^{73}, 250^{+91}, 251^{94}$, $359^{20}, 362^{31}, 383^{74}, 385^{80}, 519$

Schlobach, Jochen $29^{32}, 30^{38,40,41,43}, 31^{47}$, 50, $32^{56}, 47^{58,59}, 519$

Schlör, Joachim $356^{4}, 360^{26}, 371^{46}, 373^{54}$, $387^{87}, 389^{93}, 519$

Schmausser-Strauss, Beatrix $\quad 19^{38}, 519$

Schmitt, Stefan $387^{89}, 519$

Schneiders, Werner $34^{69}, 41^{24}, 52^{82}, 520$

Scholz, Dieter $406^{157}, 441^{286}, 520$

Schöne, Wolfgang $11^{45}, 520$

Schrenk, Klaus $51^{75}, 55^{95}, 56^{101}, 388^{89}$, $488,498,520$

Schulte, Hans H. $460^{340}, 510$

Schwartz, Vanessa R. $263^{118}, 520$

Schwarz, Heinrich $\quad 65^{12}, 185^{149}, 520$

Schwarz, Sigismond 496

Schweppenhäuser, Hermann $336^{142}$

Scotti, Roland $337^{147,148}, 505,520$

Sébillot, Amédée $245^{+73}, 520$

Sec, P. 479

Seebacher, Jacques 494

Seidel, Katrin $19^{86}, 41^{24}, 42^{25}, 201^{217}, 520$

Seitter, Walter $332^{132}, 520$

Serman, William $94^{44}, 107^{99}, 520$

Seurat, Georges $356^{5}, 377^{62}, 404^{151}, 438^{276}$

Shapiro, Anne-Louise $391^{99,101,520}$

Shapiro, Theda $404^{151}, 520$

Sharpe, William $356^{5}, 520$ 
Shikes, Ralph $79^{69}, 81^{77}, 123^{144}, 302^{40}$, $407^{163}, 448^{299}, 520$

Siebe, Michaele $83,83^{84}, 520$

Sièyes, Emmanuel $52^{78}, 495$

Signac, Paul $80,114^{118}, 404^{151}, 407,438^{276}$, 485

Silverman, Deborah $242^{59}, 245^{68}, 520$

Silverman, Willa Z. $30^{136}, 520$

Simmen, Jeannot $\quad 4^{18}, 49^{65}, 457^{333}, 520$

Simone, Franco $30^{43}, 520$

Simonin, Michel $30^{43}, 495$

Sirinelli, Jean-François $1^{3}, 14^{62}, 63^{1}, 515$, $517,518,520$

Sluijters, Georges Joseph von 480

Sohn, Andreas $92^{36}, 523$

Solo $22^{95}, 82^{80}, 158^{56}, 477,478,479,480$, $481,482,483,484,485,530$

Sonn, Richard D. $279^{179}, 496^{112}$, $398^{120,124}, 404^{150}, 435^{258}, 521$

Sorel, Georges $410^{+170}, 495$

Soubise, Camille 394

Souza, Arunda d' $113^{115}, 511$

Sparn, Walter $3^{13}, 521$

Spector, Jack $429^{227}, 448^{300}, 449^{303}, 521$

Spinner, Kaspar $11^{45}, 521$

Spoer 478

Springer, Annemarie $396^{112}, 406^{159}$, $407^{163}, 521$

Stahel, Urs $232^{25}, 521$

Starobinski, Jean $42^{26,29}, 521$

Steen, Jürgen $240^{48}, 521$

Steenhuyse, D. $398^{122.123}, 408^{168}, 521$

Steinlen, Théophile Alexandre $79^{69}$, $80^{+74}, 82,120^{+139.141}, 121,122,124$, $127,270^{+151}, 271,272^{155.156}, 276,277$, $278^{+173.175}, 280,281,284,290^{+14}, 291$, $292,298,302^{+41}, 303,304^{+47}, 313,314$, $319,340,341^{156}, 346^{+179}, 347,350,352$, $378,385^{+81.82}, 386,394^{107}, 405^{+155}$, $429^{+230}, 430,434^{250}, 438,439,444^{292}, 446$, $447,448^{298}, 449^{+302,306}, 450,451,456$, $457^{+332 / 334}, 458,459^{336}, 484,485,487,489$, $490,491,492,493,496$

Sternberger, Dolf $427^{+224}, 521$

Stierle, Karlheinz $30^{39}, 245^{70}, 456^{329}, 521$

St. James, Ashley $80^{72}, 521$

Stoll, André $59^{121}, 157^{47}, 265^{133}, 501,502$

Stone, Judith F. $422^{215}, 521$

Stop $114,115,144,145,147,151,153$, $156^{44}, 157^{50}, 311,485,487,488$

Stuart, Robert $289^{9}, 302^{105}, 410^{171}, 426^{220}$, 521

Studeny, Christophe $\quad 451^{307}, 521$
Stuke, Horst $51^{74}, 521$

Sunyer y Miro, Joaquin 307,485

Sutcliffe, Anthony $389^{98}, 404^{151}, 506,520$, 521

Swart, Koenraad Walter $128^{164}, 212^{260}$, $213^{264}, 216^{267}, 521$

Taboureau, Georges 485

Talkenberger, Heike $\quad 6^{27}, 521$

Tapdur 482

Tartakowsky, Danielle $217^{273}, 264^{123}$, $295^{25}, 452^{308}, 521$

Tartuffe 197

Tatin-Gourier, Jean-Jacques $61^{127}, 521$

Tel 478

Terrou, Fernand $\quad 68^{31}, 72^{46}, 521$

Tervarent, Guy de $324^{102}, 521$

Tessié du Motay, Cyprian $177^{116}$

Theinhardt, Markéta $338^{151}, 339^{153}, 522$

Thézy, Marie de $357^{9}, 358^{15}, 359^{21}, 360^{25}$, $371^{49}, 373^{52}, 522$

Thiers, Adolphe $57^{+110}, 58,85^{5}, 89^{+17}$, $108^{105}, 117^{125}, 124,125,126,141,151^{+31}$. $153,156^{43}, 463$

Thomas, Edith $129^{175}, 130^{181}, 522$

Thomas von Aquin 26

Thombs, Robert $89^{17}, 106^{96}, 107^{104}, 503$, 522

Thomson, Richard $79^{71}, 274^{162}, 378^{62}, 522$

Thöne, Albrecht $473^{15}, 522$

Thüsen, Joachim von der $45^{47}, 46^{49.53 .54}$, $47^{56,61}, 522$

Tillier, Bertrand $\quad 9^{38}, 13^{52}, 63^{2}, 64^{3}, 72^{43}$, $76^{52}, 77^{57}, 81^{79}, 82^{82}, 88^{11}, 100^{69}, 104^{81}$, $105^{85,87}, 112^{111}, 114^{120}, 126^{158.160}, 146^{13}$, $163^{77}, 216^{268}, 288^{4}, 444^{292}, 522$

Timms, Edward $355^{2}, 504$

Tiret-Bognet, Georges $368^{43}, 369,485$, 491

Titzmann, Michael $18^{80}, 522$

Toulouse-Lautrec, Henri de $392^{107}, 485$

Tournier, Maurice $398^{122.123}, 522$

Traeger, Jörg $41^{21}, 132^{182}, 522$

Traviès de Villiers, Charles-Joseph $49^{67}$, 55

Trinquier, Hervé $468^{6}, 495$

Trousson, Raymond $44^{38}, 522$

Tuan, Yi-Fu $355^{2}, 522$

Tulard, Jean $53^{88}, 54^{91}, 55^{99}, 57^{109}, 58^{116}$, $156^{46}, 176^{106}, 522$

Turgot, Anne Robert Jacques, Baron de

l'Aulne $44^{38}, 238$

Tury, Henri $207^{240}, 485$ 
Uelsberg, Gabriele $120^{139}, 522$

Ulbricht, Justus H. $\quad 460^{340}, 522$

Umbach, Maiken $46^{55}, 522$

Uzanne, Octave $158^{53}, 495$

Uzès $82,347^{179}, 482$

Vaillant, Auguste $398^{126}$

Val 485

Valdès/Val d'Es (auch: Es/d'Es) 485

Valio 483

Vallès, Jules $93^{41}$

Vallotton, Félix $80^{+72}, 302^{40}, 319,328$, $338^{+149}, 353^{+194}, 485,504,506,521$

Valveranne, Louis Joseph Marie Denis 394, 423, 426, 485, 491

Vandenbroeck, P. $\quad 65^{12}, 185^{149}, 522$

Van Veen, Otto $99^{61}, 495$

Varb 478

Varga, Lucie $30^{39}, 522$

Varias, Alexander $396^{112}, 397^{118}, 435^{256,259}$, $437^{274}, 523$

Vendredi 485

Verdon, Jean $356^{4}, 523$

Verhaeren, Émile $405^{+152}, 406^{156}$

Vermersch, Eugène $107^{102}, 399^{129}$, 495

Vernillat, France $140^{202}, 494$

Veuillot $58^{111}, 141^{2}$

Viellard, Fabien 482

Vignola, Amédée $200^{216}, 485$

Villon-Lechner, Alice $40^{12}, 232^{25}, 511$, 512,521

Vincent, Catherine $19^{86}, 523$

Da Vinci, Leonardo $192^{183}$

Viremaître, Charles 496

Viviani, René $203^{222,225}, 204,205,412$, $414^{188,191}, 420^{+210}, 422^{+211}, 467,488$

Voltaire $52^{+83}, 53,135^{190}, 191^{+175,176}$, $192^{+181}, 193^{+184}, 212^{259}, 225^{4}$

Volx, Denis 485

Voss, Jürgen $100^{64}, 523$

Vovelle, Michel $8,37^{1}, 51^{75}, 61^{127}, 98^{55}$, 498,523

Wachenheim, Pierre $185^{149}, 523$

Wagner, Fritz $48^{63}, 523$

Wagniart, Jean-François $448^{298}, 523$

Waldeck-Rousseau, Pierre 206, 207, $313^{72}$

Walter, Eric $191^{175}, 508$

Walter, François $440^{279}, 505$
Walther, Elisabeth $18^{79}, 523$

Warner, Marina $240^{50}, 523$

Warnke, Martin $44^{36}, 523$

Watelet, Jean $21^{89}, 23^{96}, 63^{1}, 523$

Weber, Hermann $92^{36}, 523$

Wedemeyer, Bernd $436^{264}, 523$

Weigl, Eberhard $44^{38}, 238^{47}, 523$

Weisberg, Gabriel P. $\quad 189^{169}, 270^{152}, 382^{72}$, $396^{112}, 504,515,521,523$

Wendland, Bernd $192^{183}, 523$

Werrett, Simon $232^{24}, 523$

Willette, Adolphe Léon $118,119,120$, $124^{+151}, 126,251,252,253,271,272^{+155}$, $290^{14}, 340 ; 346,351,394,418^{+204}, 419$, $427,428,429^{227}, 442^{289}, 485,487,489$, 491, 492, 493

Williams, Rosalind H. $\quad 120^{140}, 177^{113}$, $383^{74}, 523$

Williot, Jean-Pierre $\quad 20^{87}, 137^{196}$, $176^{108,110}, 177^{113,115,116}, 178^{119}, 179^{124,126}$ $183^{136}, 357^{9,11}, 358^{14}, 360^{26}, 364^{33}, 365^{37}$, $383^{74}, 523$

Wilson, Colette $231^{17}, 523$

Wilson, Stephen $301^{37}, 341^{156}, 523$

Winands, Klaus $457^{333}, 509$

Winock, Michel $340^{155}, 523$

Wirsching, Andreas $92^{36}, 100^{64}, 523$

Wittgenstein, Ludwig $6^{24}, 523$

Wolbert, Klaus $454^{323}, 524$

Wolfschmidt, Gudrun $4^{18}, 49^{67}, 524$

Wülfing, Wulf $4^{16}, 516$

Wuyts, A. G. $341,342,344,403^{+144}, 485$, 490

Yriarte, Charles 497

Zagrodzki, Christophe $406^{155}, 500$

Zajonc, Arthur $4^{18}, 524$

Zapperi, Roberto $52^{78}, 495$

Zbinden, Rolf $92^{113}, 125^{153}, 499$

Zed 485

Zelle, Carsten $34^{70}, 524$

Zisly, Henri 435

Zo d'Axa (Alphonse Gallaud de la Pérouse) 290, 292, 293

Zola, Émile $289^{+8}, 304,306^{+55}, 307^{+59}$, $311^{+67}, 312^{68,71}, 313^{72}, 314^{+73}, 321^{+94}, 327$, $330^{+126}, 338,394,404^{149}, 434^{+249,250,251,253}$, 464,495

Zut 482

Zut, A. 480 


\subsection{Medien}

L'Action quotidienne $1^{+1}, 195^{+193}, 196^{+196}$, $197^{199}, 279^{+176,179}, 352^{190}, 414^{192}, 488,497$

Album des Temps nouveaux $185^{148}$, $339^{+152}, 353,385,405,406,448^{299}, 496$

Almanach de la Calotte $197^{203}, 496$

Almanach du Charivari $167,440^{280}, 488$, 496

Almanach du libertaire $172,488,497$

Almanach du Père peinard $276^{171}, 405^{152}$, 446

L'Assiette au beurre 23, 77 $55,186^{152}$, $205^{+232}, 208^{246}, 271^{+153,154}, 274,280^{182}$, $281,282^{+183}, 283,346^{+177.179}, 347,348^{+181}$. $183,349,351^{187}, 352,353^{192}, 365^{+38}, 366$, $367^{+40}, 373^{54}, 376^{+60}, 379^{+64,65,66}, 381^{68}$, $382^{72}, 385^{+82}, 386^{+84}, 401^{140}, 408,409$, $420,421,423,431,477,478,479,480$, $481,482,483,484,485,489,490,491$, $496,505,509,513,520$

L'Aube $66^{16}, 392^{107}, 496$

L'Aurore $66^{16}, 289^{8}, 306^{55}, 312,313$, $314^{+73.74}, 330,338,394,490,497$

La Bataille $156^{13}, 444^{292}, 445,483,492$, 497

La Bataille syndicaliste $284,285,452^{+311}$, 489,498

Le Ça ira $397^{+115.117,398,498}$

La Calotte 23, 197, 198204, 205, 352 $2^{191}, 483$, 484,496

Le Canard sauvage $23,457,496$

La Caricature (1830-1835) 4967, 54, $55^{95.97}, 56^{102,103.105}, 57^{107}, 99,146^{12}$, $223^{291}, 232^{27}$

La Caricature (1880-1914) 2293, 23, $161^{+63}, 368^{+43}, 369,477,478,479,480$, $481,482,483,484,485,491,496$

Le Chambard socialiste $23,82,120^{+139,141}$ $121^{142,143}, 276,277,340,341^{156}, 346^{179}$, $387^{86}, 394^{107}, 439^{230}, 430,444^{292}, 446,447$, $449,450,451,452,479,485,487,489$, 492,496

La Charge $23,85^{1}, 99^{+59}, 132^{182}, 482,496$. 524

Le Charivari $23,54,55^{95}, 57^{108}, 58^{+111,112 .}$ $113,115,117,59+119,120,60^{+122}, 61+129,130,131$ $66^{16} 67^{+20}, 68^{+29,30}, 85^{+3.5}, 86,87^{7}, 88^{13.16}$, $95,102,103,105,110^{+110}, 111,114^{121}$, $115,123^{145}, 137^{197}, 144^{9}, 11,145,147$,
$147^{14.16,17}, 148,150^{25}, 151^{+30}, 153^{34}$, $156^{44}, 157^{48,50,51}, 158^{52}, 163^{76}, 164,165$, $166^{81}, 167,168^{88}, 174^{102}, 175,177,179^{122}$, $180^{131}, 181,182,185^{150}, 186^{+158}, 192$, $194^{+190}, 232^{28}, 240^{48}, 241^{54}, 256^{+103}$, $265^{133}, 311^{64}, 346^{177}, 347^{180}, 367^{41}, 368$, $370,371^{47,48}, 372,373^{50}, 374,381^{+70}$, $388^{89}, 452,470^{10}, 478,479,480,481,482$, $483,484,485,487,488,491,496$

Le Chat noir $22^{93}, 23,82,347^{179}, 362^{29}$, $389^{+95}, 390,479,481,491,496$

Le Cocorico $22^{93}, 23,193^{+186}, 488,496$

La Comédie politique $23,82,267^{142}$, $295^{+23}, 296,311^{65}, 325^{106}, 329,478,483$, 490,496

Le Courrier français $23,73^{+49 / 50}, 74,75$, $81,119,248,251,252,273^{+160}, 279^{+180}$, $280,362,363,371^{45}, 383^{+75}, 384^{+77}$, $395^{110}, 401,301^{141}, 402,427,428,429^{227}$, $478,479,480,481,482,483,484,485$, $497,489,490,491,492,496$

Le Cri 477

Le Cri-Cri 23, 157, 478, 482, 496

Le Cri de Paris $22^{94}, 23,276^{+171}, 317^{85}$, $319,338^{149}, 479,480,481,482,496$

La Diane $262^{116}$

Le Don Quichotte $21^{90}, 22^{93}, 23,64^{+9}$, $159,160,163,191,387^{89}, 480,496$

L'Écho de Paris $317^{85}, 412^{182}, 478,480$, 481, 498

L'Éclipse $\quad 32^{93}, 23,60^{124}, 65,66^{+16.17,18}$, $87,151^{27.28}, 152,154,155,198^{204}, 205^{232}$, $226^{+11}, 227,478,479,480,481,482,483$, $484,488,489,496$

L'État naturel et la part du prolétaire dans la civilisation $436^{267}, 498$

L'Événement $203^{223}, 332^{+131}, 414^{192}, 481$, 498

La Feuille $22^{93}, 23,290^{+14,15}, 293^{+19}$, $302^{+41}, 303,304,479,482,485,489,490$, 496

Le Fifre $290^{14}$

Le Figaro $141^{2}, 311^{+67}, 312^{+68.72}, 326^{+109}$, $330^{125}, 332^{+129}, 412^{+182.188}, 478,481,485$, 498

Le Fouet $23,313^{72}, 479,497$

La France anti-cléricale $23,188^{159}, 497$ 


\section{La Gifle 477}

Gil Blas $304^{44}, 480,497,498$

Gil Blas illustré $23,270,280,378,378^{63}$, $385^{82}, 448^{298}, 477,479,480,482,485,497$

Le Grelot 23, 64, 66 ${ }^{16}, 94^{+43}, 108,109$, $123^{+146}, 132^{182}, 141^{+1}, 142,143,151^{+29}$, $153,154^{38,40}, 161,163^{+77}, 186^{154}, 187$, $189^{+172}, 190,234^{32}, 254^{102}, 255,311$, $313^{72}, 328^{120}, 334^{133}, 368,417,478,479$, $482,483,487,488,489,497$

Guguess! 482

L'Humanité $127^{+161}, 412^{182}, 449^{+306}$, $459^{+337}, 485,498$

Les Humoristes 485

\section{L'Intransigeant $\quad 78,200$}

\section{La Jeune Garde $262^{116}$}

La Journée $\quad 158^{+55,56}, 159^{57}, 480,482,498$

Le Libertaire $23,171,289,290^{12}, 295,297$, $341,342,344,397^{118}, 400^{134}, 403^{+144}, 434$, $435^{254}, 448^{301}, 460^{341}, 485,490,497$

La Liberté 179, 498

La Libre Parole illustrée 23, 82, 137, 479, 485,497

La Lune $65^{+14,15}, 66^{+16}, 70,231^{21}, 479$, 480

La Lune rousse $22^{93}, 23,66^{+18}, 180^{+132}$, $226^{+9,12}, 228,229,231^{+21}, 234,235^{34}$, $242^{57}, 478,489,497$

\section{La Minerve $53^{+84,85}$}

Le Mirliton 24, 438+277, 439, 446, 492, 497

Le Monde illustré $128,130^{+179}, 229^{+14,16}$, $230,231^{19}, 235^{+35}, 238^{+46}, 239,246^{+74}$, $479,483,489,497$

Le Monde nouveau 457

Le Monde parisien $130^{181}, 497$

Le Monde pour rire 124, 497

Le Nain jaune 51

Le Naturien $22^{93}, 435^{260}, 436^{262}, 437^{267}$, 498, 513

Nebelspalter $205^{237}$

Le Nouveau Père Duchêne $399^{129}$

La Nouvelle Lune $22^{93}, 24,130,131$, $236^{42}, 259^{+111}, 260,482,483,487,489$, 497

Pages folles 24, 392, 393, 423, 425, 426, $480,481,491,497$
Le Pèlerin $1^{+2}, 23^{96}, 24,82,128,129^{167-174}$ $139,198^{+208}, 199,200,201^{218,220}, 202$, $203^{+228}, 204,205^{229,230,231}, 209,210,211$, $216^{266}, 217^{+271}, 218^{+276,278}, 219,224^{297}$, $240^{48}, 248^{+85}, 249,379^{+67}, 380,397^{116}$, $422^{211}, 478,481,488,489,491,497$

Le Père Duchêne 399129

Le Père peinard $24,82,140^{+202}, 274164$, $279,399^{+129}, 400^{+137,139}, 441^{+285}, 443,483$, $492,497,513$

Le Pétard $22^{93}, 24,66^{16}, 180^{+133}, 192$, $365^{39}, 482,497$

Le Petit Bleu de Paris $78,315,317^{84}, 318$, $320,411^{180}, 412^{188}, 479,481,484,485$, 490, 498

La Petite Lune 24, 66, 158+54, 480, 497

Le Peuple souverain $77^{56}, 149,150^{25}, 498$

Le Pied de nez 485

Le Pierrot $290^{14}, 485$

Le Pilori 24, 161, 275, 478, 483, 485, 497

Psst!... 24, 82 $82,288^{4}, 312^{71}, 478,480,497$, 522

Le Radical $208^{247}, 498$

La Révolte $399,400,408^{168}$

La Révolution politique et sociale $89^{+21}$, 498

Rigoletto 482

Le Rire $24,77^{55}, 78,81,203^{225}, 205^{+235,236}$ $206,208,209,269^{+147}, 375^{59}, 383^{76}, 385^{83}$, $394^{109}, 412,413,416,418,419,477,478$, $479,480,481,482,483,484,485,488$, 491,497

Le Ruy Blas $22^{93}, 24,388^{89}, 431^{+236}, 485$, 497

La Semaine anti-cléricale 169, 170, 173, $181^{135}, 189^{165}, 196^{197}, 398,498$

Le Siècle $78,330^{+127}, 334^{134}, 341,411^{178}$, 498

Le Sifflet (1872-1888) $24,66^{16}, 309^{+62}$ 310. 483. 490. 497

Le Sifflet (1898-1899) 24. 66 ${ }^{16}, 82^{82}, 288^{4}$, $299,300,302,312^{71}, 316^{+76,81}, 317^{+85}$, $319^{91,}, 92,325,328,331,332^{128}, 333$, $334^{135,136,138,478,479,480,481,490,497}$

La Silhouette (1829-1830) 54

La Silhouette (1880-1914) 24, 6932, 82, $242,243,244^{65}, 256,258,259^{112}, 261$, $263^{+121}, 270,312^{71}, 388^{89}, 482,483,484$, 489,497

Le Socialiste $452^{+310}, 498$

Le Soleil 65 
Le Témoin $\quad 24,415,480,481,484,491$, 497

Les Temps nouveaux $240^{50}, 339,343^{163}$, $400,437,441^{284}, 479,481,483,484,485$, 497

Le Triboulet $24,67^{+24}, 68,79^{67}, 132^{+184,186}$, $133,134,135^{+193}, 136,137^{+198}, 138,200$ $216,203^{226}, 207^{+239,240}, 211,212,214$, $217^{+272.275}, 218^{281}, 220^{+284}, 221,222^{287.288}$.

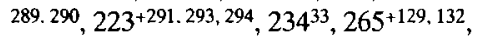

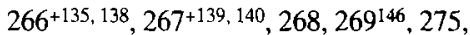
$288^{7}, 309^{+63}, 321,322,375^{+57}, 414^{191}, 422$, $477,478,480,482,483,484,485,487$, $488,489,490,497$

La Vache enragée 485

La Vie moderne $113^{116}, 479,497$

La Voix du peuple 411, 414, 483, 491, 498

La Volonté $327^{+115}, 481,498$ 
